\title{
Knocking-out matrix metalloproteinase-13 exacerbates rotator cuff muscle fatty infiltration
}

\author{
Xuhui Liu1, 2 \\ Bharat Ravishankar² \\ Anne Ning ${ }^{1,2}$ \\ Mengyao Liu $^{2}$ \\ Hubert T. Kim 1,2 \\ Brian T. Feeley ${ }^{1,2}$
}

1 San Francisco Veterans Affairs Medical Center, Department of Veterans Affairs, San Francisco, USA

2 Department of Orthopaedic Surgery, University of California, San Francisco, USA

\author{
Corresponding author: \\ Xuhui Liu \\ San Francisco Veterans Affairs Medical Center \\ 1700 Owens Street, Room 364 \\ 94153 San Francisco, CA, USA \\ E-mail: liux@orthosurg.ucsf.edu
}

\section{Summary}

Introduction: Rotator cuff (RC) tears are common tendon injuries. Clinically, both muscle atrophy and fatty infiltration have generally been attributed to poor functional outcomes. Matrix metalloproteinase-13 plays a crucial role in extracellular matrix remodeling in many physiological and pathological processes. Nevertheless, its role in rotator cuff muscle atrophy and fatty infiltration remains unknown. The purpose of this study is to define the functional role of MMP13 in rotator cuff muscle atrophy and fatty infiltration using a mouse RC tears model.

Materials and methods: Unilateral complete supraspinatus and infraspinatus tendon transection and suprascapular nerve transection was performed on nine of MMP-13 (-/-) knockout and nine of MMP-13 (+/+) wildtype mice at 3 months old. Mice were sacrificed 6 weeks after surgery. Supraspinatus (SS) and infraspinatus (IS) muscles were harvested for histology and gene expression analysis with RT-PCR.

Results: Six weeks after RC surgery, no significant difference in muscle atrophy and fibrosis between MMP-13 knockout and wild type mice was observed. However, there was a significant increase in the amount of fatty infiltration in MMP-13 knockout mice compared to the wild types. Muscles from MMP-13 knockout mice have significantly higher expression of fatty infiltration related genes.
Discussion: Results from this study suggest that MMP- 13 plays a crucial role in rotator cuff muscle fatty degeneration. This novel finding suggests a new molecular mechanism that governs RC muscle FI and MMP-13 may serve as a target for therapeutics to treat muscle $\mathrm{FI}$ after RC tears.

KEY WORDS: rotator cuff tear, matrix metalloproteinase13, muscle, fatty infiltration.

\section{Introduction}

Rotator cuff $(\mathrm{RC})$ tears are one of the most common injuries seen by orthopaedic surgeons ${ }^{1}$. Small and medium sized tears are amenable to surgical repair, while massive and chronic tears often cause significant impairment of shoulder function. While healing rates are often dependent on tendon to bone healing, outcomes following surgical repair are predicated on both tendon healing and improved muscle function. The latter is generally governed by muscle atrophy and fatty infiltration $(\mathrm{FI})^{2-5}$.

Prior works have shown that various matrix metalloproteinases (MMPs) link to pathophysiological changes in skeletal muscle 6 . MMPs are ECM proteases known to affect a variety of cellular functions including cell proliferation, migration, and differentiation. MMP-1, 2, 3, 9 and other ECM remodeling molecules have been demonstrated to be associated with rotator cuff tears ${ }^{7-10}$. In our previous work, we have observed significantly increased expression of MMP13 in rotator cuff muscle after tendon injury in a sheep model ${ }^{11}$. However, the functionality of MMP-13 in this context remains undefined.

MMP-13 has been shown to affect cellular migration, thus serving as a marker for metastatic cancers ${ }^{12}$. Upregulation of its activity in myoblast cell lines ${ }^{13}$ suggests that it may affect the migration of satellite cells. Muscle fatty infiltration is believed be caused by fat progenitors migrating into the muscle following, resulting the buildup of fat ${ }^{14}$. Though other works suggest resident muscle multipotent progenitor cells are the source of fat cells in muscle, it is believed that cues from the extracellular matrix and paracrine signaling pathways play a role in mediating this event ${ }^{15,16}$.

The purpose of this study was to examine the role of MMP-13 in rotator cuff muscle $\mathrm{FI}$ in following a massive rotator cuff tear. We employed MMP-13 null mice in a previously established rodent $\mathrm{RC}$ tears model ${ }^{17}$, 18. We found that MMP-13 null mice have significantly worsen muscle FI compared to wildtype mice. This 
result suggests that MMP-13 plays a critical role in rotator cuff muscle $\mathrm{FI}$ after massive RC tears. MMP13 may serve as a potential pharmacological target in the treating rotator cuff muscle $\mathrm{Fl}$ in the future.

\section{Materials and methods}

\section{Animal Surgery}

MMP-13 knockout mice was received from the Krane Laboratory in Harvard School of Medicine as a generous gift. Heterozygous MMP-13 (+/-) knockout mice was used as breeders and homozygous MMP-13 (-/-) knockout mice and their littermate wildtype MMP-13 $(+/+)$ mice were used in the experiment. In total, nine of MMP-13 (-/-) and nine of MMP-13 (+/+) mice at 3 months old underwent unilateral massive rotator cuff (RC) tear surgery. Unilateral complete supraspinatus and infraspinatus tendon transection and suprascapular nerve transection was performed on mice as described previously ${ }^{17,18}$. A sham surgery, in which the tendons and nerve were exposed but not transected, was performed on the contralateral side. We strictly followed International Guide for the Care and Use of Laboratory Animals ${ }^{19}$ as well as our local Institutional Animal Care and Use Committee (IACUC) protocol during all procedures and handling of the animals.

\section{Muscle Harvest}

Mice were sacrificed 6 weeks after surgery. Supraspinatus (SS) and infraspinatus (IS) muscles were harvested and the remaining tendon and scar tissue were removed at the muscle/tendon junction. The wet weights of muscles were measured immediately. Subsequently, supraspinatus muscles from four MMP-13 (-/-) and four MMP-13 (+/+) mice was prepared for cryogen sectioning while muscles from the rest five MMP-13 (-/-) and five MMP-13 (+/+) mice homogenized for protein and RNA extraction.

\section{Histology}

Supraspinatus muscles from both shoulders were harvested and flash frozen in liquid nitrogen-cooled 2 -methylbutane. The muscles were then cryosectioned at a thickness of $10 \mu \mathrm{m}$ at muscle belly. A Masson's trichrome stain was performed to detect the relative collagen content in extracted muscle samples with Masson's Trichrome 2000 Stain Kit (American MasterTech Inc., Lodi, CA) following a protocol provided by the manufacturer. An Oil-Red-O stain was performed to gauge levels of fat accumulation in harvested muscle using a protocol from our previous studies ${ }^{17}$. In brief, frozen sections were fixed in $3.7 \%$ formaldehyde for one hour and then stained with a working Oil-Red-O solution 30 minutes. Slides were then mounted in a $10 \%$ glycerol solution. Collagenous tissue from the Trichrome Stain and fat seen in the Oil-Red-O stain were quantified using Image $\mathrm{J}$ $(\mathrm{NIH})$. The area of collagen deposition or fat was divided by the total area of the section.

\section{Reverse transcriptase-polymerase chain reaction (RT-PCR)}

Total RNA was isolated using Trizol reagent (Invitrogen, Inc., Carlsbad, CA) from supraspinatus muscles according to manufacturer's instructions. cDNA was synthesized using Transcriptor First Strand cDNA Synthesis Kit (Roche Applied Bioscience, Indianapolis, IN). Real-time PCR was performed to quantify the expression of various fatty infiltration markers using SYBR Green Detection and an Applied Biosystems Prism 7900HT detection system (Applied Biosystems, Inc., Foster City, CA) with the following primers: PPARI (forward) 5'-GCATGGTGCCTTCGCTGA-3', and (reverse) 5'-TGGCATCTCTGTGTCAACCATG; SREBP-1 (forward) 5'- AACCTCATCCGCCACCTG3', and (reverse) 5'-GTAGACAACAGCCGCATCC-3'; Fatty acid synthase (FASN) (forward) 5'-GCTGGCATTCGTGATGGAGTCGT-3', and (reverse) 5'AGGCCACCAGTGATGTAACTCT-3'; GAPDH: (forward) 5'-TGCACCACCAACTGCTTAG-3', and (reverse) 5'-GGATGCAGGGATGATGTTC-3'. The gene expression level was normalized to the internal control of GAPDH. Fold changes were calculated using $\Delta \Delta-\mathrm{C}_{\mathrm{T}}$.

\section{Statistical Analysis}

T-test was used for data analysis between wild type and knockout muscle. Significant difference was considered when $\mathrm{p}<0.05$.

\section{Results}

\section{Significant decrease in muscle wet weight follow- ing surgery}

Six weeks following tendon and nerve transection surgery, there was a highly significant $(p<0.01)$ decrease in muscle wet weight of harvested supraspinatus muscles in both wild-type and knockout mice. However, no significant differences were observed between MMP-13 knockout and wildtype animals (Fig. 1).

\section{No significant differences in muscle fibrosis between MMP-13 (-/-) and MMP-13 (+/+) mice after surgery}

Collagenous accumulation was carefully quantified using ImageJ from the Trichrome staining slides. In both wildtype and MMP-13 knockout mice, there is a visible increase of collagenous tissue in supraspinatus muscles after surgery. However, there was no significant difference seen between MMP-12 knockout and wildtype mice ( $p=0.61)$ (Fig. 2 e).

\section{MMP-13 (-/-) mice have greater muscle FI com- pared to MMP-13 (+/+) mice}

Both MMP-13 knockout and wild-type operated muscles contained a higher amount of fat compared to the sham sides (Fig. 3 a, c). However, MMP-13 knockout muscles demonstrated a significantly higher amount of fat following surgery than the wild-type mice (Fig. 3 b, d) $(p<0.01$, Fig. 3 e). 


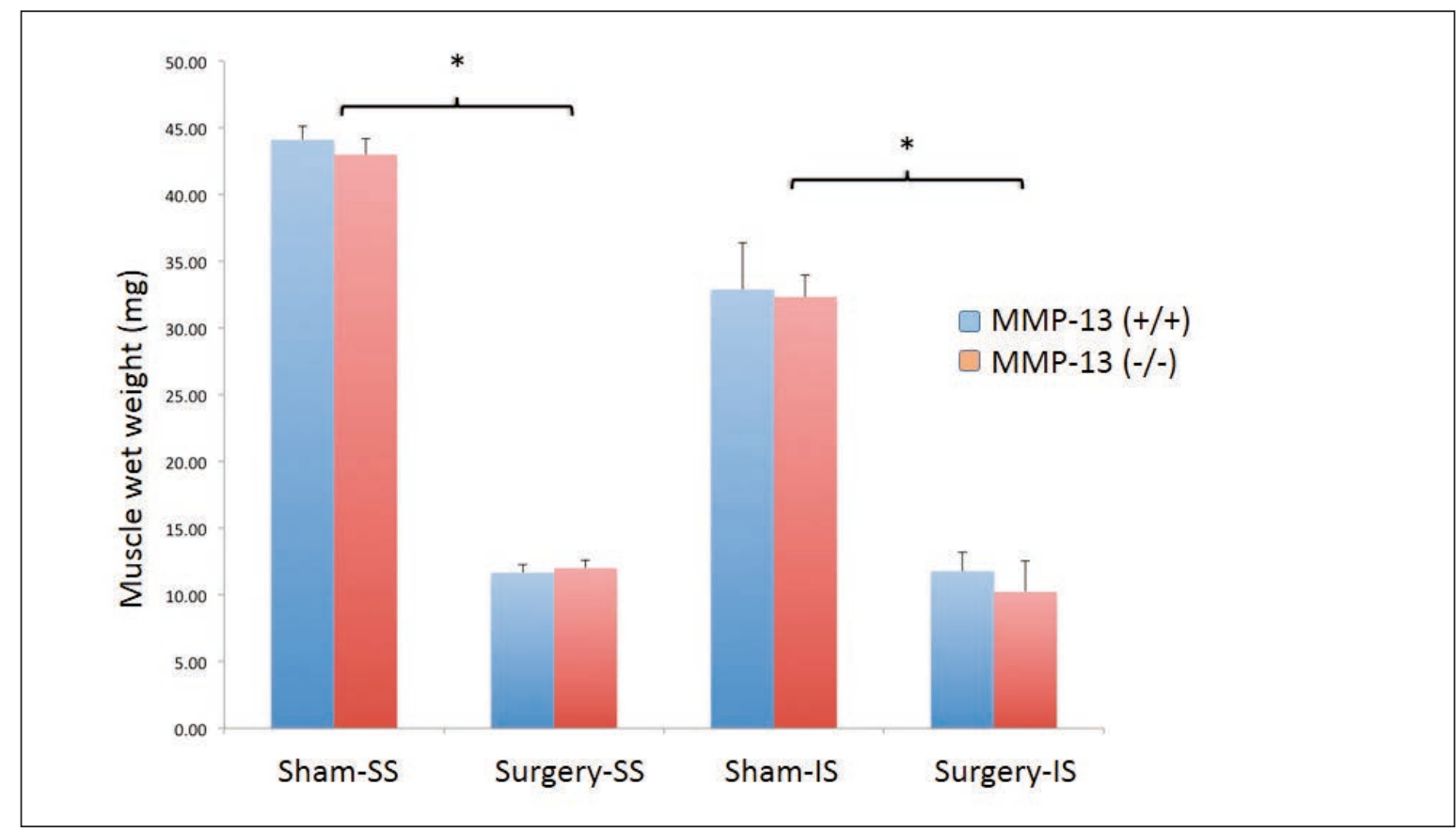

Figure 1. No difference of muscle atrophy between MMP-13 knockout (KO) and wildtype (WT) mice. Six weeks following tendon and nerve transection surgery, there was a highly significant $\left({ }^{*}, p<0.01\right)$ decrease in muscle wet weight of harvested supraspinatus muscles in both wild-type and knockout mice. However, no significant differences were observed between MMP-13 (-/-) and wildtype MMP-13 (+/+) mice.

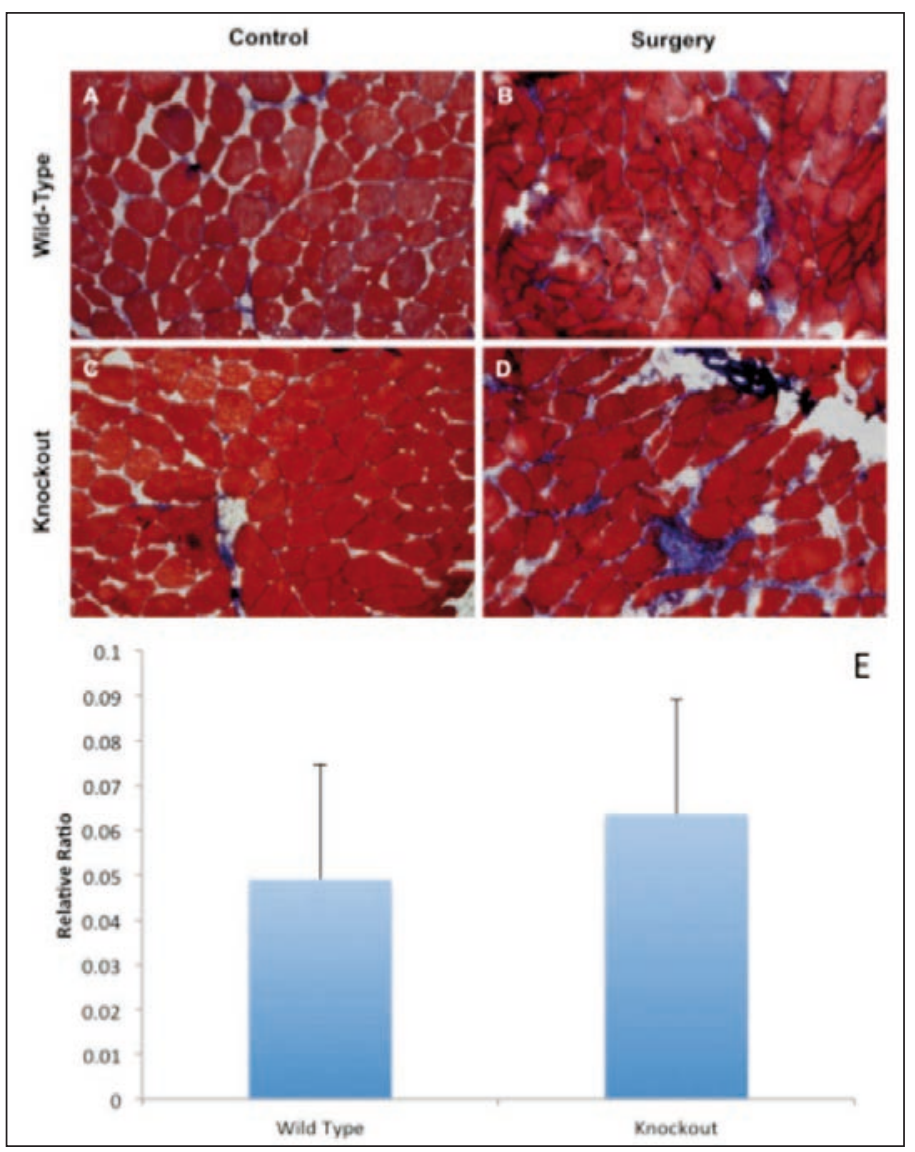

Figure 2 A-E. No difference of muscle fibrosis between MMP-13 knockout (KO) and wildtype (WT) mice. Sections of supraspinatus muscle from all mice groups $(n=4)$ were analyzed for collagenous connective tissue using Masson Trichrome staining and photographed at 20x. Groups were separated into: A) wild-type sham side, B) wild-type surgery side, C) knockout sham side, and D) knockout surgery side, showing increased accumulation of collagen deposition in supraspinatus muscle at surgery side in both MMP-13 knockout and wildtype mice. However, no difference was seen in between MMP13 knockout and wildtype mice $(E)(p=0.61)$. 


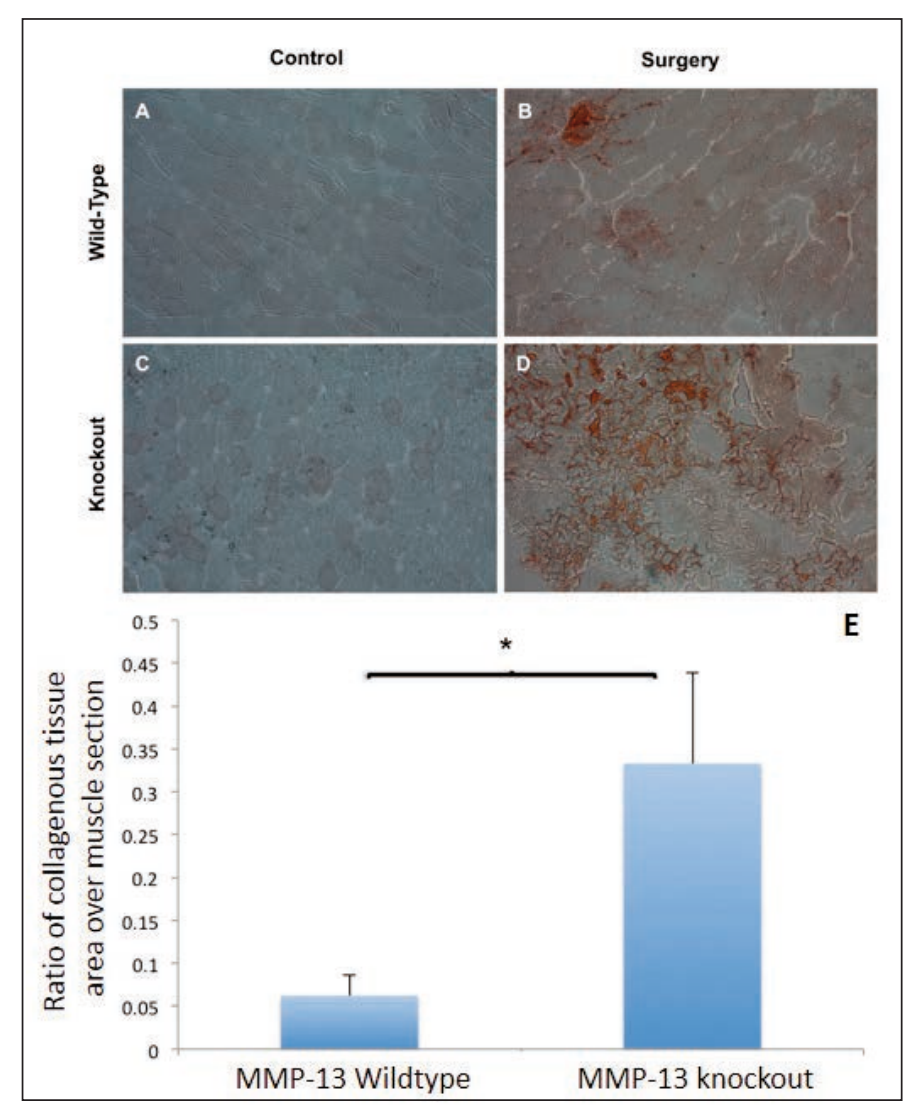

Figure 3 A-D. Increase fatty infiltration in MMP-13 knockout mice. Sections of supraspinatus muscle from both groups $(n=4)$ were assessed for the amount of fatty infiltration and photographed at a 10X magnification. Groups were separated into: A) wild-type sham side, B) wild-type surgery side, C) knockout sham side, and D) knockout surgery side. Fat was quantified using ImageJ through a relative area ratio and the mean values of these ratios graphed $(E)\left(^{*}, p<0.01\right)$.

\section{Adipogenic gene expression is up-regulated in MMP-13 knockout mice}

Six weeks after surgery, gene expression level of PPARg increased $5.08 \pm 1.12$ fold, SREBP-1 increased $9.71 \pm 3.30$ fold and FASN increased $18.6 \pm$ 4.51 fold in supraspinatus muscle at surgery side in
MMP-13 (-/-) mice compared to MMP-13 (+/+) mice $(p<0.05$, Fig. 4). No difference of expression levels of those genes was seen in supraspinatus muscle at the sham side between MMP-13 (-/-) and MMP-13 (+/+) mice (data not shown).

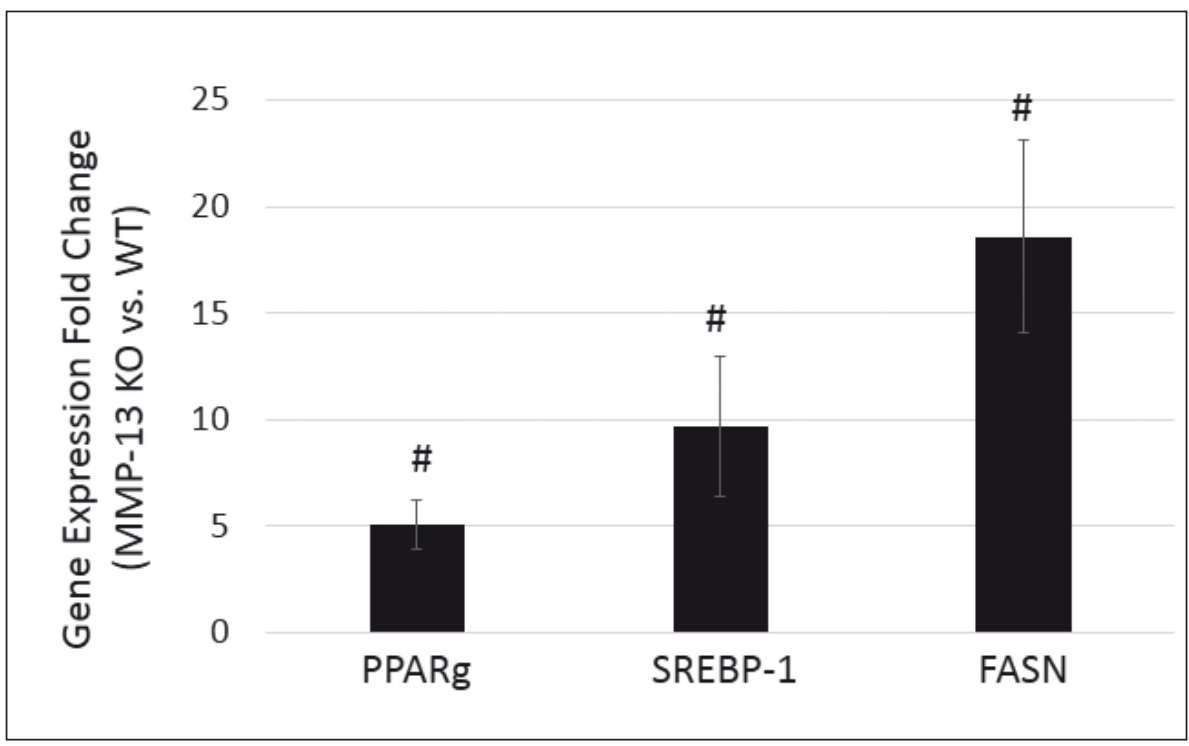

Figure 4. Fold change of expression level of adipogenic markers in MMP13 knockout mice compared to wildtype mice. Expression level of PPARY, SREBP-1 and FASN significantly increased at six weeks after TT+DN surgery in supraspinatus muscles in MMP-13 (-/-) mice compared to MMP $-13(+/+)$ mice $(\#, p<0.05)$. 


\section{Discussion}

Results from this study demonstrated that MMP-13 knockout mice have significantly increased FI in rotator cuff muscle after tendon and nerve injury evidenced from both a histological and biochemical perspective. This novel data suggests that MMP-13 plays an important role in RC muscle $\mathrm{FI}$ and may potentially serve as a new pharmacological target in treating $\mathrm{RC}$ muscle fatty degeneration.

The MMP-13 gene is comprised of 10 exons and 9 introns spanning $12.5 \mathrm{~kb}$ on chromosome $11^{20}$. There is a TIE (TGF $\beta$ inhibitory element) site in MMP-13 promoter. There are also two other sites resemble consensus sequences for the Activin-response element and Smad binding element ${ }^{20}$. Previous studies have reported that $\mathrm{TGFb}$ regulates MMP-13 expression in various tissues. BMPs are a subgroup of the TGF $\beta$ family. Similar to TGF $\beta$, BMPs engage serine/threonine kinase receptors that phosphorylate Smad1/5/8, which governs target gene expression. A recent study showed that BMP-14 inhibits MMP-13 expression in chondrocytes ${ }^{21}$. In our previous work, we have shown significantly increased TGF $\beta^{22}$ and $\mathrm{BMP}^{23}$ signaling in rotator cuff muscles after massive tendon tears, suggesting TGF $\beta / B M P$ signaling may be involved in rotator cuff muscle fatty degeneration through regulating MMP-13 expression. Future works are needed to define the role of TGF $\beta / B M P$ signaling in regulating MMP-13 in RC muscles.

Uezemi et al. ${ }^{24}$ showed that cells PDGFRa ${ }^{+}$muscle fibro-adipogenic progenitor cells (FAPs) are responsible for adipogenic differentiation in the muscle after chemical-induced muscle injury. In our previous study, we have shown that FAPs are the major cellular source of adipocytes seen in RC muscles ${ }^{25}$. Lei et al. ${ }^{13}$ found that MMP-13 had an influence on the migration of satellite cells. But the role of MMP-13 on FAPs proliferation, migration and differentiation remains unknown. Our next future work will be to investigate the role of MMP-13 in regulating FAP adipogenesis in vitro and in vivo.

There are some limitations in this study that ought to be addressed. First of all, MMP-13 knockout mice adopted in this study is a global knockout model. A tissue specific knockout of MMP-13 in the muscle could provide an even more accurate representation of its effects on muscle pathology following an RCT. Future experiments, both in vivo and in vitro, will employ this technique to use as a comparison to the knockout study. Second, only one time point was adopted in this study due to limited number of knockout animals. We believe this is sufficient of the first step to investigate the role of MMP-13 in RC muscle $\mathrm{Fl}$. More time points could be included in future work for better define the mechanism of MMP-13 regulating RC muscle fatty degeneration. Though some PCR was conducted, the conclusion form this study was largely based on histology analysis. Though this may be sufficient for the first step, more sophisticated biochemical and biophysical experiments and analysis are needed in future to better understand how MMP13 regulating $\mathrm{RC}$ muscle $\mathrm{FI}$.
Our study clearly demonstrates that MMP-13 plays a role in muscle pathology changes following massive tendon injury. This novel finding suggests a new molecular mechanism that governs RC muscle $\mathrm{FI}$ and MMP-13 may serve as a target for therapeutics to treat muscle $\mathrm{FI}$ after $\mathrm{RC}$ tears.

\section{Conflict of interest}

The Authors have no conflict of interest.

\section{Acknowledgements}

This work was supported by the Orthopaedic Research and Education Foundation, UCSF Core Center for Musculoskeletal Biology and Medicine (NIH 1P30AR066262-01) and VA BLR\&D Merit review grant (1 I01 BX002680-01A2). We thank Dr. Stephen M. Krane (Harvard Medical School) for his kind gift of MMP-13 knockout mice. We also thank Ms. Nai Chen Chang and Mr. Erik Kramer for their technique support in histology analysis. No Authors has any conflicts of interest to disclose.

\section{References}

1. Oliva F, Piccirilli E, Bossa M, Via AG, Colombo A, Chillemi C, et al. I.S.Mu.L.T - Rotator Cuff Tears Guidelines. Muscles Ligaments Tendons J. 2015 Oct-Dec;5(4):227-263.

2. Zanetti M, Gerber C, Hodler J. Quantitative assessment of the muscles of the rotator cuff with magnetic resonance imaging. Invest Radiol. 1998 Mar;33(3):163-170.

3. Goutallier D, Postel JM, Bernageau J, Lavau L, Voisin MC. Fatty muscle degeneration in cuff ruptures. Pre- and postoperative evaluation by CT scan. Clin Orthop Relat Res. 1994 Jul;(304):78-83.

4. Coleman SH, Fealy S, Ehteshami JR, MacGillivray JD, Altchek DW, Warren RF, et al. Chronic rotator cuff injury and repair model in sheep. J Bone Joint Surg Am. [Comparative Study Research Support, Non-U.S. Gov't]. 2003 Dec;85A(12):2391-2402.

5. Safran O, Derwin KA, Powell K, lannotti JP. Changes in rotator cuff muscle volume, fat content, and passive mechanics after chronic detachment in a canine model. J Bone Joint Surg Am. 2005 Dec;87(12):2662-2670.

6. Chen X, Li Y. Role of matrix metalloproteinases in skeletal muscle: migration, differentiation, regeneration and fibrosis. Cell Adh Migr. 2009 Oct-Dec;3(4):337-341.

7. Jacob J, Eisemon E, Sheibani-Rad S, Patel A, Jacob T, Choueka J. Matrix metalloproteinase levels as a marker for rotator cuff tears. Orthopedics. 2012 Apr;35(4):e474-478.

8. Bedi A, Kovacevic D, Hettrich C, Gulotta LV, Ehteshami JR, Warren RF, et al. The effect of matrix metalloproteinase inhibition on tendon-to-bone healing in a rotator cuff repair model. Journal of shoulder and elbow surgery/American Shoulder and Elbow Surgeons [et al.]. 2010 Apr;19(3):384-391.

9. Castagna A, Cesari E, Garofalo R, Gigante A, Conti M, Markopoulos N, et al. Matrix metalloproteases and their inhibitors are altered in torn rotator cuff tendons, but also in the macroscopically and histologically intact portion of those tendons. Muscles Ligaments Tendons J. 2013 Jul;3(3):132138. 
10. Del Buono A, Oliva F, Osti L, Maffulli N. Metalloproteases and tendinopathy. Muscles Ligaments Tendons J. 2013 Jan;3(1): 51-57.

11. Luan T, Liu X, Easley JT, Ravishankar B, Puttlitz C, Feeley BT. Muscle atrophy and fatty infiltration after an acute rotator cuff repair in a sheep model. Muscles Ligaments Tendons J. 2015 Apr-Jun;5(2):106-112.

12. Vihinen $P$, Kahari VM. Matrix metalloproteinases in cancer: prognostic markers and therapeutic targets. International journal of cancer Journal international du cancer. 2002 May;10;99 (2):157-166.

13. Lei H, Leong D, Smith LR, Barton ER. Matrix metalloproteinase 13 is a new contributor to skeletal muscle regeneration and critical for myoblast migration. American journal of physiology Cell physiology. 2013 Sep;305(5):C529-538.

14. Pannerec A, Formicola L, Besson V, Marazzi G, Sassoon DA. Defining skeletal muscle resident progenitors and their cell fate potentials. Development (Cambridge, England). 2013 Jul;140 (14):2879-2891.

15. Desai VD, Hsia HC, Schwarzbauer JE. Reversible modulation of myofibroblast differentiation in adipose-derived mesenchymal stem cells. PloS one. 2014 Jan 23;9(1):e86865.

16. Sullivan KE, Quinn KP, Tang KM, Georgakoudi I, Black LD, 3rd. Extracellular matrix remodeling following myocardial infarction influences the therapeutic potential of mesenchymal stem cells. Stem cell research \& therapy. 2014 Jan 24;5(1):14.

17. Joshi SK, Liu X, Samagh SP, Lovett DH, Bodine SC, Kim HT, et al. mTOR regulates fatty infiltration through SREBP-1 and PPARgamma after a combined massive rotator cuff tear and suprascapular nerve injury in rats. J Orthop Res. 2013 May;31 (5):724-730.
18. Liu X, Laron D, Natsuhara K, Manzano G, Kim HT, Feeley BT. A mouse model of massive rotator cuff tears. J Bone Joint Surg Am. 2012 Apr 4;94(7):e41.

19. Padulo J, Oliva F, Frizziero A, Maffulli N. Muscles, Ligaments and Tendons Journal - Basic principles and recommendations in clinical and field science research: 2016 update. MLTJ. 2016;6(1):1-5.

20. Pendas AM, Balbin M, Llano E, Jimenez MG, Lopez-Otin C. Structural analysis and promoter characterization of the human collagenase-3 gene (MMP13). Genomics. 1997 Mar 1;40(2):222-233

21. Enochson L, Stenberg J, Brittberg M, Lindahl A. GDF5 reduces MMP13 expression in human chondrocytes via DKK1 mediated canonical Wnt signaling inhibition. Osteoarthritis Cartilage. 2014 Apr;22(4):566-577.

22. Liu X, Joshi SK, Ravishankar B, Kim HT, Feeley BT. Upregulation of transforming growth factor-beta signaling in a rat model of rotator cuff tears. J Shoulder Elbow Surg. 2014 May 27.

23. Liu X, Joshi S, Ravishankar B, Laron D, Kim HT, Feeley BT. Bone morphogenetic protein signaling in rotator cuff muscle atrophy and fatty infiltration. Muscles Ligaments Tendons $\mathrm{J}$. 2015 Apr-Jun;5(2):113-119.

24. Uezumi A, Fukada S, Yamamoto N, Takeda S, Tsuchida K. Mesenchymal progenitors distinct from satellite cells contribute to ectopic fat cell formation in skeletal muscle. Nature cell biology. 2010 Feb;12(2):143-152.

25. Liu X, Ning AY, Chang NC, Kim H, Nissenson R, Wang L, et al. Investigating the cellular origin of rotator cuff muscle fatty infiltration and fibrosis after injury. Muscles Ligaments Tendons $\mathrm{J}$. 2016 Jan-Mar;6(1):6-15. 